\title{
Desenvolvimento e caracterização de snack de milho extrusado com adição de
}

\section{farinha de ora-pro-nóbis}

\author{
Development and characterization of extruded corn snack with addition of ora-pro-nobis flour \\ Desarrollo y caracterización de snack de maíz extruido con adición de harina de ora-pro-nobis
}

Recebido: 10/02/2021 | Revisado: 20/02/2021 | Aceito: 25/02/2021 | Publicado: 03/03/2021

\author{
Maria Fernanda Francelin \\ ORCID: https://orcid.org/0000-0003-4499-4265 \\ Universidade Estadual de Maringá, Brasil \\ E-mail: mferfrancelin@hotmail.com \\ Leonardo Martins Machado \\ ORCID: https://orcid.org/0000-0003-2705-5848 \\ Universidade Estadual de Maringá, Brasil \\ E-mail: leomm.martins@outlook.com \\ Denise de Moraes Batista da Silva \\ ORCID: https://orcid.org/0000-0002-5698-3654 \\ Universidade Estadual de Maringá, Brasil \\ E-mail: denise_mbsilva@hotmail.com \\ Eloize da Silva Alves \\ ORCID: https://orcid.org/0000-0002-3340-8374 \\ Universidade Estadual de Maringá, Brasil \\ E-mail: eloizeetaus@gmail.com \\ Rosane Marina Peralta \\ ORCID: https://orcid.org/0000-0001-5749-0723 \\ Universidade Estadual de Maringá, Brasil \\ E-mail: rmperalta@uem.br \\ Silvio Claudio Costa \\ ORCID: https://orcid.org/0000-0001-9002-3000 \\ Universidade Estadual de Maringá, Brasil \\ E-mail: sccosta@uem.br \\ Antônio Roberto Giriboni Monteiro \\ ORCID: https://orcid.org/0000-0003-1894-0765 \\ Universidade Estadual de Maringá, Brasil \\ E-mail: argmonteiro@uem.br
}

\begin{abstract}
Resumo
A ora-pro-nóbis faz parte de um grupo de hortaliças não convencionais que não são encontradas em cardápios usualmente devido à falta de conhecimento da população a respeito de seus benefícios nutricionais. No entanto, as plantas alimentícias não convencionais se destacam como alimentos funcionais devido a vitaminas, antioxidantes, fibras, proteína e sais minerais encontradas em plantas, como é o caso da ora-pro-nóbis. O presente trabalho teve o objetivo de elaborar um snack de milho extrusado com adição de farinha de folhas de ora-pro-nóbis (OPN) da espécie Peréskia grandifolia Haw (PG), visando aumentar o valor nutritivo deste produto e avaliar sua aceitação sensorial perante a avaliadores não treinados. A colheita, higienização e desidratação, foram efetuadas para a obtenção da farinha das folhas. Posteriormente, esta farinha foi submetida a análises bromatológicas de rotina como umidade, proteínas, lipídios, cinzas, ferro e fibras tendo resultados próximos aos encontrados na literatura. Com a farinha das folhas foram desenvolvidas duas formulações com adição de 10 e $20 \%$ de farinha de folhas respectivamente, juntamente com um padrão de composição de $100 \%$ grits de milho. Os produtos prontos foram submetidos as mesmas análises bromatológicas anteriormente citadas, além de uma análise sensorial de aceitação por escala Hedônica com provadores não treinados. O suplemento desenvolvido de folhas de ora -pro-nóbis apresentou alto valor biológico em proteínas em fibras além de uma boa aceitação, mostrando-se viável sua utilização.
\end{abstract}

Palavras-chave: Peréskia grandifolia Haw; Composição centesimal; Análise sensorial; Extrusão; Salgadinho.

\begin{abstract}
The ora-pro-nobis is part of a group of unconventional vegetables that are not usually found on menus due to the population's lack of knowledge about its nutritional benefits. However, unconventional food plants stand out as functional foods due to vitamins, antioxidants, fibers, protein and minerals found in plants, as is the case of ora-pronobis. The present work had the main intention to elaborate an extruded corn snack with addition of ora-pro-nobis leaf meal (OPN) of the species Peréskia grandifolia Haw (PG), aiming to increase the nutritional value of this product and
\end{abstract}


evaluate its sensorial acceptance to untrained evaluators. Harvesting, cleaning and dehydration were carried out to obtain the leaf flour. Subsequently, this flour was subjected to routine bromatological analyzes such as moisture, proteins, lipids, ash, iron and fibers, with results close to those found in the literature. With the leaf flour, two formulations were developed with the addition of 10 and $20 \%$ leaf flour respectively, together with a composition standard of $100 \%$ corn grits. The finished products were subjected to the same bromatological analyzes previously mentioned, in addition to a sensory analysis of acceptance by Hedonic scale with untrained tasters. The supplement developed from ora-pro-nobis leaves showed high biological value in fiber proteins in addition to good acceptance, proving viable its use.

Keywords: Peréskia grandifolia Haw; Centesimal composition; Sensory analysis; Extrusion; Snack.

\section{Resumen}

El ora-pro-nobis forma parte de un grupo de verduras no convencionales que no suelen encontrarse en los menús debido al desconocimiento de la población sobre sus beneficios nutricionales. Sin embargo, las plantas alimenticias no convencionales se destacan como alimentos funcionales debido a las vitaminas, antioxidantes, fibras, proteínas y minerales que se encuentran en las plantas, como es el caso de ora-pro-nobis. El presente trabajo tuvo como objetivo principal la elaboración de un snack de maíz extruido con adición de harina de hoja de ora-pro-nobis (OPN) de la especie Peréskia grandifolia Haw (PG), con el objetivo de incrementar el valor nutricional de este producto y evaluar su sensibilidad sensorial. aceptación antes a evaluadores no capacitados. Se llevó a cabo la recolección, limpieza y deshidratación para obtener la harina de hoja. Posteriormente, esta harina fue sometida a análisis bromatológicos rutinarios como humedad, proteínas, lípidos, cenizas, hierro y fibras, con resultados cercanos a los encontrados en la literatura. Con la harina de hoja se desarrollaron dos formulaciones con la adición de 10 y $20 \%$ de harina de hoja respectivamente, junto con una composición estándar de $100 \%$ sémola de maíz. Los productos terminados fueron sometidos a los mismos análisis bromatológicos mencionados anteriormente, además de un análisis sensorial de aceptación por escala hedónica con catadores no capacitados. El suplemento desarrollado a partir de hojas de ora-pronobis mostró un alto valor biológico en proteínas de fibra además de una buena aceptación, resultando viable su uso.

Palabras clave: Peréskia grandifolia Haw; Composición centesimal; Análisis sensorial; Extrusión; Bocadillo.

\section{Introdução}

Com o passar dos anos houve um aumento populacional e juntamente com ele, o aumento do consumo dos recursos naturais existentes, sendo estes utilizados conforme a cultura e disponibilidade em cada localização do globo terrestre. Contudo, com a exploração das terras através do mundo, algumas plantas foram mais difundidas e assim consideradas como alimentos do uso cotidiano. Outras espécies foram deixadas de lado e passaram a ter seu consumo limitado a povos regionais, estas plantas então, passaram a ser consideradas como alimentos não convencionais. O termo plantas alimentícias não convencionais (PANC) foi criado em 2007 pelo Biólogo e Professor Valdely Ferreira Kinupp e refere-se a todas as plantas que possuem uma ou mais partes comestíveis, sendo elas espontâneas ou cultivadas, nativas ou exóticas que não estão incluídas em nosso cardápio cotidiano. Existem no Brasil pelo menos 3 mil espécies de plantas alimentícias com ocorrência conhecida no país. Estima-se que pelo menos $10 \%$ da flora nativa do Brasil (4 a 5 mil espécies de plantas) seja alimentícia (Kelen et al., 2015).

Algumas destas, são utilizadas regionalmente com o intuito de aumentar a diversidade das refeições. A Ora-pro-nóbis (Pereskia grandifolia Haw - PG), planta nativa dos trópicos, uma espécie de cacto, comumente encontrado na região nordeste no país, é considerada uma destas espécies utilizadas na alimentação (Rosa \& Souza, 2003). Autores a tempos já identificaram que esta planta possui um alto teor de proteínas e fibras em suas folhas, assim como a ausência de toxicidade (Almeida Filho \& Cambraia, 1974; Dayrell, 1977; Butterworth \& Wallace, 2005).

Com tantos atrativos nutricionais as características desta planta têm chamado a atenção de muitos pesquisadores devido a sua adaptabilidade e baixo custo de produção (Dias, Durigan, \& Guimarães, 2018). A Ora-pro-nóbis pode ser considerada, de acordo com a RDC n ${ }^{\circ}$ 54/2012 (Brasil, 2012), um alimento funcional por seu alto teor de proteínas (25\% aproximadamente) de alta digestibilidade (80\%), sendo esta a principal característica de alvo como uma fonte para a melhoria do quadro da insegurança alimentar de muitas famílias de baixa renda, além de ser uma fonte de nutrientes para públicos 
diversos caso a mesma seja utilizada como matéria prima no desenvolvimento de novos produtos com aceitabilidade consagrada no paladar populacional como é o caso dos snacks.

O Brasil por se apresentar como um país de dimensões continentais, possui em seu território uma vasta diferenciação de classes sociais, apresentando uma grande taxa de pessoas que possuem deficiências nutricionais graves, mesmo estas tendo acesso a alimentação, ainda optam por alimentos de baixo valor nutricional em função da alimentação pouco variada e de baixo custo (Dias, Durigan, \& Guimarães, 2018).

Em seu estudo no estado de Minas Gerais, Almeida \& Corrêa (2012) pode perceber que a planta é comumente cultivada nas residências das famílias de menor renda, porém 20,83\% não a consome com frequência, pois muitos desconhecem seu potencial e/ou possuem vergonha, as demais consideram o sabor da planta muito forte, mesmo quando associado no preparo de alimentos como em omeletes ou outros pratos típicos, logo, esta é mais uma evidência para que a implementação do desenvolvimento de um produto diferenciado utilizando a planta se posta em prática.

Além destas pesquisas referentes a planta em si, pode-se observar também que a última década de estudos da planta, se mostram como grande maioria as publicações a respeito da Pereskia ssp, seja qual for a espécie, os estudos estão voltados para área farmacêutica, demonstrando um maior interesse por suas propriedades medicinais, e uma lacuna em estudos, o que indica uma oportunidade de aprofundamento de estudo. Sendo assim, para o consumo deste alimento a sua aceitação ao paladar populacional, além de sua conservação para a ampla distribuição, de forma a manter suas características positivas de consumo (Souza, 2013).

Uma alternativa a solução desta problemática, é o processo de extrusão, método de produção de alimentos versátil, prático e de baixo custo (Cheng \& Friis, 2010). O processo trabalha com modificações com base na umidade, pressão, atrito e aquecimento, permitindo a gelatinização do amido a desnaturação da proteína, inativação de parte de taninos, diminuindo a quantidade de materiais antinutricionais e aumentando o valor biológico (Horvath, \& Greenberg, 1989) favorecendo a disponibilidade dos micronutrientes presentes nos alimentos, mantendo-o seu valor nutricional disponível quanto consumido (Chen, Wei, \& Zhang, 2011).

Para este ramo da indústria as previsões são promissoras, pois um estudo da Mintel (2018) mostra que os consumidores estão aumentando os períodos fora de casa e o consumo de snacks para saborearem é forma de lanches rápidos entre as refeições. Contudo a vertente na mudança nos hábitos alimentares da população que sem mostrado nos últimos anos, a preferência por lanches saudáveis, fez com que este mercado encontrasse formas de inovação para que o mesmo continue sendo prático, porém com seu valor nutricional atraente ao consumo.

No ramo de tecnologia de alimentos uma das principais formas de conservação se dá pela desidratação, a qual previne a deterioração e resultando em um novo produto para a população (Soares et al., 2001, Topuz, Gokoğlu, Jouppila, \& Kirjoranta, 2017). Portanto, o aumento do consumo da ora-pro-nobis, poderá ser feito caso possamos utilizar destas tecnologias para entregar um alimento versátil, de fácil consumo e que satisfaça o gosto de seus consumidores, sem deixar de lado suas características benéficas (Berwig et al., 2017; Dischsen, Monteiro, Fukuda, \& Marques, 2013).

Assim o objetivo deste trabalho foi desenvolver um snack com seu valor nutricional melhorado através da adição da farinha das folhas de ora-pro-nóbis (OPN) (da espécie Pereskia grandifolia Haw - PG), nas quantidades de adição de 10 e $20 \%$, assim como testar avaliar sua aceitabilidade por análise sensorial por escala hedônica com provadores não treinados.

\section{Metodologia}

O método cientifico selecionado para a pesquisa realizada foi um estudo de natureza quantitativa (Pereira, Shitsuka, Pereira, \& Shitsuka, 2018). 


\subsection{Coleta das amostras e obtenção da farinha}

As folhas de ora-pro-nobis (OPN) da espécie Pereskia granflora Haw (PG), foram coletadas no viveiro de mudas MUDI (Latitude 24 graus, 11 minutos e 06 segundos e Longitude 53 graus, 01 minuto e 40 segundos W-GR) da Universidade Estadual de Maringá, no mês de Abril de 2019, tendo o cuidado de proceder a colheita após 5 dias consecutivos sem chuvas.

As folhas de OPN coletadas foram levadas ao laboratório, pesadas, lavadas em solução clorada (100 ppm) e enxaguadas em água corrente, deixadas secar em estufa com circulação de ar forçado (TE-394, Tecnal) a $105{ }^{\circ} \mathrm{C}$ até obter peso constante.

As folhas desidratadas de OPN foram trituradas em moinho de facas, passadas em peneira mesh 40 e armazenadas em potes com tampas de fechamento hermético e protegidas da luz até o momento da análise.

\subsection{Composição centesimal}

As determinações feitas foram: extrato etéreo determinado pelo método de extrator continuo tipo Soxhlet, proteína bruta foi dosada pelo método Kjeldahl, utilizando o fator de conversão 6,25 ( $\mathrm{N}$ x 6,25), cinzas foram obtidas pela incineração $\left(550^{\circ} \mathrm{C}\right)$ em forno tipo mufla, Fibras brutas, fibras em detergente neutro e detergente ácido, sendo todas metodologias segundo métodos da AOAC (2005). Todas as análises foram realizadas em triplicata, tanto na farinha das folhas de OPN quanto nos snacks produzidos.

\subsection{Extrusão}

Foram processados 500 gramas de cada formulação, todas empregando grits de milho 1515 (Nutrimilho) nas quantidades apresentadas na tabela 1 .

Tabela 1. Formulações dos snacks extrusados nos três tratamentos.

\begin{tabular}{ccc}
\hline Amostra & \% grits de milho & \% de farinha de folhas \\
\hline Padrão (CP) & 100 & 10 \\
Formulação 1 (C10) & 90 & 20 \\
Formulação 2 (C20) & 80 & \\
\hline
\end{tabular}

\footnotetext{
Quantidades adicionadas de grits de milho e farinha de folhas de OPN em cada formulação, em ambas formulações houve uma adição de $3 \%$ de água.

Fonte: Autores (2020).
}

As amostras prontas foram pré-condicionadas a uma umidade de 3\%, antes do processo, que ocorreu de forma constante, sem que houvesse alteração nos parâmetros da extrusora. A extrusão foi efetuada no equipamento IMBRA RX50 (INBRAMAQ, Ribeirão Preto, SP, Brasil), com rosca única de $50 \mathrm{~mm}$ de diâmetro e $200 \mathrm{~mm}$ de comprimento. A matriz utilizada possuía dois furos de $3 \mathrm{~mm}$ de diâmetro e os parâmetros foram fixados com a amperagem do motor em 20 A e a alimentação do sistema em $15 \mathrm{~g} / \mathrm{s}$. O corte foi realizado a $120 \mathrm{rpm}$.

Posteriormente, o produto final extrusado foi homogeneizado e seco em tambor rotativo a $60{ }^{\circ} \mathrm{C}$. Todas as amostras foram submetidas ao processo de tambleamento para adição de aromatizantes e salga, contendo $4 \%$ do mix de ervas e $2 \%$ de cloreto de sódio.

O mix de ervas desidratadas era composto de $25 \%$ de salsa desidratada, $15 \%$ de orégano desidratada, $10 \%$ de cebolinha desidratada e 50\% cloreto de sódio, os ingredientes foram triturados e homogeneizados. 


\subsection{Análise sensorial}

A análise sensorial foi realizada no Laboratório de Análises do Departamento de Alimentos Engenharia na Universidade Estadual de Maringá, utilizando 100 provadores não treinados com idade entre 18 e 27 anos, sendo 78 do sexo feminino e 22 do sexo masculino.

O protocolo experimental foi aprovado pela pesquisa Comitê de Ética da Universidade Estadual de Maringá (registro $\mathrm{n}^{\circ}$ CAAE 18718013.3.0000.0104). Amostras de aproximadamente 5,0 g (15 unidades) foram servidas em copos de plástico descartáveis, previamente codificados aleatoriamente e o teste foi feito com cada provador avaliando as três amostras individualmente e em sequência.

Cada provador recebeu três amostras, identificadas por códigos aleatórios, e utilizou a escala hedônica, estruturada de nove pontos, variando entre 1 (desgostei extremamente) e 9 (gostei extremamente) para avaliar os atributos cor, sabor, aroma, textura e aparência global de cada amostra (Monteiro, 2005).

\subsection{Análise estatística}

Os dados obtidos foram avaliados estatisticamente a partir da análise de variância (ANOVA), com posterior análise das médias pelo teste de Tukey a 5\% de probabilidade com auxílio do programa SISVAR (Ferreira, 2011).

\section{Resultados e Discussão}

Os resultados das análises centesimais dos três tratamentos realizados na produção de snacks extrusados estão apresentados na Tabela 2.

Tabela 2. Resultados das análises centesimais dos snacks extrusados produzidos.

\begin{tabular}{cccc}
\hline Análises (\%) & CP & C10 & C20 \\
Umidade & $6,40 \pm 0,24^{\mathrm{a}}$ & $6,27 \pm 0,04^{\mathrm{a}}$ & $5,46 \pm 0,22^{\mathrm{b}}$ \\
Cinzas & $1,80 \pm 0,16^{\mathrm{a}}$ & $1,99 \pm 0,04^{\mathrm{a}}$ & $2,02 \pm 0,12^{\mathrm{a}}$ \\
Lipídeos & $8,61 \pm 0,07^{\mathrm{c}}$ & $10,53 \pm 0,31^{\mathrm{b}}$ & $13,83 \pm 0,08^{\mathrm{a}}$ \\
Proteínas & $8,31 \pm 0,95^{\mathrm{b}}$ & $10,60 \pm 0,58^{\mathrm{a}}$ & $11,67 \pm 0,85^{\mathrm{a}}$ \\
Carboidratos & $74,00 \pm 0,32^{\mathrm{a}}$ & $69,38 \pm 0,05^{\mathrm{b}}$ & $63,11 \pm 0,42^{\mathrm{c}}$ \\
Fibra Bruta & $0,88 \pm 0,48^{\mathrm{b}}$ & $1,23 \pm 0,08^{\mathrm{b}}$ & $3,91 \pm 0,01^{\mathrm{a}}$ \\
& & & \\
Fibra Detergente Neutro & $63,59 \pm 0,32^{\mathrm{a}}$ & $21,39 \pm 1,99^{\mathrm{b}}$ & $13,38 \pm 0,49^{\mathrm{c}}$ \\
& & & \\
Fibras Detergente Ácido & $1,98 \pm 0,34^{\mathrm{b}}$ & $2,30 \pm 0,18^{\mathrm{b}}$ & $5,75 \pm 0,47^{\mathrm{a}}$ \\
& & &
\end{tabular}

Todos valores demonstrados por análises em base seca. CP: amostra controle 100\% grits de milho; C10: 10\% de farinha de folhas de OPN; C20: 20\% de farinha de folhas de OPN. Médias seguidas pela mesma letra na linha não diferem estatisticamente entre si pelo teste de Tukey a $5 \%$ de probabilidade. Análise feita de comparação aos pares entre as amostras presentes no estudo.

Fonte: Autores (2020). 
Foi possível observar uma diminuição da umidade na amostra com inclusão de $20 \%$ de farinha de folhas de OPN, em relação ao padrão, contudo todos os valores atendem a legislação vigente da vigilância sanitária, para snack, que delimita um máximo de 14\% (Brasil, 1978). Estes dados possuem uma característica positiva, visto que este parâmetro está diretamente ligado a proliferação de microrganismos e a estabilidade química e enzimática do produto, além de afetar diretamente características sensoriais como a textura e corânica, logo para este quesito as formulações mostraram-se satisfatórias, apresentando valores inferiores ao parâmetro (Barbosa-Cánovas, Fontana, Schmidt, \& Labuza, 2007).

O teor de cinzas nos snacks teve acréscimo conforme aumentou a adição da farinha de folha nas formulações, esta é uma característica importante que merece destaque, uma vez que esta análise indica a presença de minerais que atuam no bom funcionamento do metabolismo. Kinupp (2009) que estudaram a espécie concluíram que entre as PANCS os teores de cinzas são os maiores nas ora-pro-nóbis (OPN) podendo, portanto, serem consideradas uma fonte de minerais.

Quando comparado os valores de lipídios entre as formulações, nota-se um aumento na sua porcentagem diretamente proporcional a inclusão da farinha das folhas OPN.

A Agência Nacional de Vigilância Sanitária pela RDC nº 269 (Brasil, 2005), indica que uma pessoa adulta deve ter uma ingestão diária de proteína de $50 \mathrm{~g}$, o produto desenvolvido neste estudo mostra-se uma boa fonte de proteínas, indo de encontro ao desenvolvimento de snacks mais saudáveis conforme a demanda comentada na introdução deste mesmo trabalho. Observando o valor de proteína no produto extrusado, tendo diferenças no grau de inclusão da farinha das folhas de OPN, foi perceptível que as amostras não diferem entre si, porém, diferem da amostra controle contendo apenas milho em sua formulação, onde há um aumento médio do valor de proteína encontrado no produto, resultado satisfatório denotando a melhoria no valor nutricional e a agregação das folhas de OPN como uma alternativa viável.

Para os parâmetros de carboidratos, há uma diminuição considerável de $10 \%$ entre a formulação padrão e a formulação C20. Os carboidratos são abundantes em cereais como o milho, aparecem em quantidades aproximadas de $80 \%$ de sua composição (Silva Santos et al., 2018), a sua substituição pela farinha, fez com que o resultado se apresentasse desta forma, tornando os alimentos com características nutricionais mais interessantes.

Os valores de fibras brutas das três formulações possuem diferença apenas quando a inclusão é de $20 \%$ de farinha de folhas de OPN. O teor de fibra bruta apresenta o resultado sobre as fibras insolúveis, contudo esta análise é complementada pelo valor da análise de fibras em detergente neutro (FDN), sendo assim, quando avaliado este parâmetro de FDN é possível perceber que houve uma diminuição inversamente proporcional a inclusão, mostrando que a adição da farinha das folhas de OPN diminuiu a proporção de fibra detergente neutro.

O comportamento contrário podemos perceber para fibras em detergente ácido (FDA), e em ambos os casos há diferenças entre as amostras, salvo para inclusão de 10\% para FDA. De acordo com Frankelin et al. (2018) a fração representada por celulose, hemicelulose e lignina são detectadas pela análise de FDN, esta são as fibras chamadas de insolúveis, fibras estas que interferem diretamente na absorção de nutrientes, sua diminuição possibilita a melhor biodisponibilidade de ferro e vitaminas presente nos alimentos, logo este dado nos mostra que o processo de extrusão possibilitou uma diminuição desta fração que leva melhor absorção dos nutrientes. Este dado, pode nos mostrar que, apesar do aumento da inclusão da farinha das folhas de OPN, não há aumento deste parâmetro no produto final, informando que, o processo de extrusão possui a características de modificar as fibras insolúveis, sendo este um ponto positivo, uma vez que é sabido que este parâmetro interfere diretamente de forma negativa na absorção de bionutrientes presentes na matriz das folhas.

Ainda em relação as fibras, a Portaria n 27, de 13 de janeiro de 1998 - Regulamento Técnico referente a Informação Nutricional Complementar - considera que para que um alimento seja considerado fonte de fibras, este deve conter no mínimo $3 \mathrm{~g}$ de fibras $/ 100 \mathrm{~g}$, com isso o snack C20, possui um resultado de 3,91\% de fibra bruta em sua composição, sendo, portanto, considerado um alimento fonte de fibras (Brasil, 1998). 
Durante a extrusão, o acréscimo de $10 \%$ e $20 \%$ da farinha das folhas de OPN obteve-se resultados satisfatório, pois as três formulações possuiriam características de expansão aproximadas, não afetando a qualidade e característica principal do snack, o que pode mostrar, que o processo de extrusão utilizando as inclusões analisadas de farinha das folhas torna-se viável.

O processo de extrusão ocorre sob altas temperaturas em curto tempo e é usado na indústria de alimentos para produzir produtos expandidos, assim como snacks, cereais matinais e rações, sendo assim, quaisquer mudanças nos ingredientes como açúcar, sal e fibra, podem afetar as variáveis do sistema de extrusão e as características do produto como: textura, estrutura, grau de expansão e atributos sensoriais, ou até mesmos inviabilizar o processo como um todo (Mendonça, Grossmann, \& Verhé, 2000).

Para os resultados de análise sensorial através do teste de escala Hedônica, obteve-se os dados apresentados na Tabela 3.

Tabela 3. Resultados de análise sensorial dos snacks extrusados produzidos.

\begin{tabular}{cccc}
\hline Parâmetro & CP & C10 & C20 \\
\hline Cor & $6,93 \pm 0,43^{\mathrm{a}}$ & $4,72 \pm 0,66^{\mathrm{b}}$ & $3,43 \pm 0,50^{\mathrm{c}}$ \\
Sabor & $6,94 \pm 0,49^{\mathrm{a}}$ & $5,51 \pm 0,45^{\mathrm{b}}$ & $4,07 \pm 0,46^{\mathrm{c}}$ \\
Aroma & $6,49 \pm 0,46^{\mathrm{a}}$ & $5,84 \pm 0,38^{\mathrm{b}}$ & $5,49 \pm 0,62^{\mathrm{b}}$ \\
Textura & $6,98 \pm 0,51^{\mathrm{a}}$ & $6,27 \pm 0,53^{\mathrm{b}}$ & $5,51 \pm 0,54^{\mathrm{c}}$ \\
& & & \\
Aparência Global & $7,05 \pm 0,65^{\mathrm{a}}$ & $5,49 \pm 0,58^{\mathrm{b}}$ & $4,29 \pm 0,40^{\mathrm{c}}$
\end{tabular}

CP: amostra controle 100\% grits de milho; C10: 10\% de farinha de folhas de OPN; C20: 20\% de farinha de folhas de OPN. Médias seguidas pela mesma letra na linha não diferem estatisticamente entre si pelo teste de Tukey a 5\% de probabilidade. Análise feita de comparação aos pares entre as amostras presentes no estudo.

Fonte: Autores (2020).

Os snacks obtidos através das formulações de incorporação de farinha de folhas de OPN nas proporções de 10 e $20 \%$, obtiveram de forma geral decrescentes conforme aumentou a inclusão das folhas. Apesar destes valores decrescentes foi possível verificar que até a inclusão de $10 \%$ as conceitos médios foram iguais ou superiores a 5, sendo consideradas avaliações positivas assim como os encontrados por Nascimento, Silva, Alves, \& Monteiro, (2021) em um desenvolvimento de produto extrusado.

\section{Conclusão}

A inclusão de farinha de folhas de OPN na produção de snacks extrusados de milho mostrou um acréscimo em características nutricionais do produto, entretanto impactou em uma redução nas características sensoriais conforme se aumentou a inclusão da farinha de folha de OPN nas formulações. Entretanto, os atributos sensoriais com adição de $10 \%$ de farinha de folhas de OPN ainda forma considerados positivos, evidenciando a viabilidade do emprego desta farinha de folha de OPN, uma vez que incorporação melhora o teor de fibras e proteína do snack, além de contribuir para o aumento da biodisponibilidade do ferro. Futuramente deve-se fazer uma otimização para verificar o máximo de inclusão de farinha de folha de OPN sem comprometimento sensorial, para se obter o melhor desempenho nutricional mantendo a aceitação sensorial positivo. 


\section{Referências}

Almeida Filho, J. D., Cambraia, J. (1974). Estudo do valor nutritivo do “ora-pro-nobis” (Pereskia aculeata Mill.). Revista Ceres, 21 (114), 105-111.

Almeida, M. E. F. D., Corrêa, A. D. (2012). Utilização de cactáceas do gênero Pereskia na alimentação humana em um município de Minas Gerais. Ciência Rural, 42(4), 751-756.

AOAC - Association of Official Analytical Chemists (2005). Official Methods of analysis of the Association of Official Analytical Chemists. (18th ed). Gaithersburg: AOAC.

Barbosa-Cánovas, G. V., Fontana, A. J., Schmidt, S. J., \& Labuza, T. P. (2007). Water activity in foods. Fundamentals and applications.

Brasil. (1998) Agência Nacional de Vigilância Sanitária - Anvisa. Portaria $n^{o}$ 27, de 13 de janeiro de 1998. Regulamento técnico referente à informação nutricional complementar. Diário Oficial da União, Brasília, 1998.

Brasil. (2005). Agência Nacional de Vigilância Sanitária. Resolução RDC nº 269, de 22 de setembro de 2005. Diário Oficial da União, Brasília.

Brasil. (1978). Agência Nacional de Vigilância Sanitária - ANVISA. Gerência-Geral Alimentos Resolução - CNNPA $n^{\circ} 12$, de 1978. Diário Oficial da União, Brasília.

Brasil. 2012. Ministério da Saúde. Resolução $n^{\circ}$ 54. Regulamento Técnico sobre Informação Nutricional Complementar. Diário Oficial da União, Brasília.

Butterworth, C. A., Wallace, R. S. (2005). Molecular phylogenetics of the leafy cactus genus Pereskia (Cactaceae). Systematic Botany, 30(4), 800-808.

Berwig, K., Marques, D. R., Silva, D. M. B., Mendes, M., Raniero, G., Monteiro, C. C. F., \& Monteiro, A. R. G. (2017). Texture on extruded snack: correlation between instrumental and sensory analysis. Chemical Engineering Transactions, 57, 1723-1728.

Chen, F. L., Wei, Y. M., \& Zhang, B. (2011). Chemical cross-linking and molecular aggregation of soybean protein during extrusion cooking at low and high moisture content. LWT-Food Science and Technology, 44(4), 957-962.

Cheng, H., Friis, A. (2010). Modelling extrudate expansion in a twin-screw food extrusion cooking process through dimensional analysis methodology. Food and Bioproducts Processing, 88(2-3), 188-194.

Dayrell, M. (1977). Extração e estudo do valor nutritivo de proteínas de folhas de ora-pro-nobis (Pereskia aculeata Mill). 1977. 106 f (Doctoral dissertation, Dissertação (Mestrado em Bioquímica e Imunologia) -Universidade Federal de Minas Gerais, Belo Horizonte).

Dias, R. N., Durigan, M. F. B., \& Guimarães, P. V. P. (2018). Potencial do uso da Ora-pro-nobis (Pereskiaaculeata Mill.) na segurança alimentar em comunidades em situação de risco e vulnerabilidade. In Embrapa Roraima-Artigo em anais de congresso (ALICE). In: Simpósio Brasileiro De Agropecuária Sustentável, 9.; Congresso Internacional De Agropecuária Sustentável, 6., 2018, Viçosa, MG. Ciência, tecnologia e inovação para o desenvolvimento agropecuário sustentável: anais... Viçosa, MG: Biblioteca Central, Campus UFV, 2018.

Dischsen, A. E., Monteiro, A. R. G., Fukuda, G. T., \& Marques, D. R. (2013). Development of a breakfast cereal using waste from cassava processing industry. Acta Scientiarum. Technology, 35(1), 157-161.

Ferreira, D. F. (2011). Sisvar: a computer statistical analysis system. Ciência e agrotecnologia, 35(6), 1039-1042.

Francelin, M. F., Vieira, T. F., Garcia, J. A. A., Correa, R. C. G., Monteiro, A. R. G., Bracht, A., \& Peralta, R. M. (2018). Phytochemical, nutritional and pharmacological properties of unconventional native fruits and vegetables from Brazil. Phytochemicals in vegetables: A valuable source of bioactive compounds, 442-470.

Horvath, A. O., Greenberg, LS (1989). Desenvolvimento e validação do Working Alliance Inventory. Journal of Counseling Psychology, 36 (2), 223.

Kelen, M. E. B., Nouhuys, I. S. V., Kehl, L. C., Brack, P., \& Silva, D. D. (2015). Plantas alimentícias não convencionais (PANCs): hortaliças espontâneas e nativas. Porto Alegre: UFRGS.

Kinupp, V. F. (2009). Plantas alimentícias não-convencionais (PANCs): uma riqueza negligenciada. REUNIÃO ANUAL DA SBPC, 61a, 4.

Mendonça, S., Grossmann, M. V. E., \& Verhé, R. (2000). Corn bran as a fibre source in expanded snacks. LWT-Food Science and Technology, 33(1), 2-8. Mintel. Snacks Salgados 2018 - Brasil - Março 2018. Mintel Gourp LTDA. Recuperado de http://reports.mintel.com/display/876867/

Monteiro, A. R. G. (2005). Introdução à análise sensorial de alimentos. Coleção Fundamentum, Editora da Universidade Estadual de Maringá, Maringá.

Nascimento, W. J. do; Silva, D. M. B. da; Alves, E. S.; \& Monteiro, A. R. G. (2021). Desenvolvimento e caracterização de snack extrusado a base de farinha de tapioca, e avaliação sensorial sobre intenção de compra. Research, Society and Development. v. 10, n. 2, e15310212395, 2021.

Pereira, A. S., Shitsuka, D. M., Pereira, F. J., \& Shitsuka, R. (2018). Metodologia da pesquisa científica. UFSM. https://repositorio.ufsm.br/bitstream/handle/1 /15824/Lic_Computacao_Metodologia-Pesquisa-Cientifica.pdf?sequence=1.

Rosa, S. M., Souza, L. A. (2003). Morfo-anatomia do fruto (hipanto, pericarpo e semente) em desenvolvimento de Pereskia aculeata Miller (Cactaceae. Acta Scientiarum. Biological Sciences, 25(2), 415-428.

Silva Santos, D., Monteiro, S. S., Pereira, E. M., Marini, F. S., Vasconcellos, A., \& Lima, J. F. (2018). Composição centesimal de milho Crioulo coletado em localidades do estado da Paraíba. Revista Verde de Agroecologia e Desenvolvimento Sustentável, 13(3), 308-312.

Souza, L. F. (2014). Aspectos fitotécnicos, bromatológicos e componentes bioativos de Pereskia aculeata, Pereskia grandifolia e Anredera cordifolia. 
Research, Society and Development, v. 10, n. 3, e2910312850, 2021

(CC BY 4.0) | ISSN 2525-3409 | DOI: http://dx.doi.org/10.33448/rsd-v10i3.12850

Soares, E. C., Oliveira, G. S. F. D., Maia, G. A., Monteiro, J. C. S., Silva Jr, A., \& Filho, M. D. S. D. (2001). Desidratação da polpa de acerola (Malpighia emarginata DC) pelo processo" foam-mat". Food Science and Technology, 21(2), 164-170.

Souza, M. R. D. M. (2013). Ora-pro-nobis (Pereskia aculeata Mill.) como alternativa promissora para produção de proteína: densidade de plantio e adubação nitrogenada.

Topuz, O. K., Gokoğlu, N., Jouppila, K., \& Kirjoranta, S. (2017). Development of extruded shrimp-corn snack using response surface methodology. Turkish Journal of Fisheries and Aquatic Sciences, 17(2), 333-343. 